\title{
A COMPARISON OF OCULOMOTOR AND MOTION PARALLAX CUES OF EGOCENTRIC DISTANCE
}

\author{
Walter C. Gogel and Jerome D. Tietz \\ University of California, Santa Barbara, California 93106, U.S.A.
}

(Received 11 September 1978)

\begin{abstract}
Using the head motion procedure, the apparent distance of a point of light in an otherwise dark visual field was measured under conditions in which oculomotor cues (accommodation, convergence) and absolute motion parallax were varied together and separately. It was concluded that absolute motion parallax is almost as effective a cue to distance as are oculomotor cues from monocular observation, but is not as effective as oculomotor cues from binocular observation. Evidence was also presented that the null adjustment method, used in conjunction with the head motion procedure, provides an unbiased measure of apparent distance.
\end{abstract}

In a series of articles (Gogel, 1976. 1977; Gogel and Newton, 1976; Gogel and Tietz, 1973, 1974, 1977) a new method of measuring apparent distance called the head motion procedure has been described and applied to the measurement of perceived distance from several distance cues. With the head motion procedure, the head is moved in a frontoparallel plane and judgments are obtained of the apparent motion of the stimulus object concomitant with the motion of the head. From these judgments, as will be explained, the experimenter can compute the distance of the object as perceived by the observer. The head motion procedure has several important advantages over other, more direct, methods for measuring apparent distance such as that of obtaining verbal reports of apparent distance. Unlike direct methods, with the head motion procedure the observer is unaware of the relation between his judgment and apparent distance and, therefore, cannot modify the response in an attempt to be veridical.

From the above studies, it is clear that the head motion procedure provides a useful and sensitive measure of apparent distance. In the present study several variations of this procedure are applied to the evaluation of the relative effectiveness of oculomotor and absolute motion parallax cues of egocentric distance. Also, evidence is presented supporting the assumption that the measures obtained from the head motion procedure are not only a monotonic increasing function of apparent distance but are indeed equal to apparent distance.

Figure 1 is useful in discussing a number of aspects of the head motion procedure and its assumptions. The prime notation indicates perceived (apparent) characteristics and the notation without primes indicates physical characteristics in this and the following figures. Figure IA illustrates the situation in which, as the head is moved repetitively left and right through a distance $K$ between Positions 1 and 2, a point of light physically at a distance $D$ from the observer also is moved laterally through a physical horizontal distance $h$ (between $s_{1}$ and $s_{2}$ ) concomitant with, but in a direction opposite to, the motion of the head. The visual direction between the observer and the point of light pivots around a hypothetical point at a distance $D_{p}$ from the observer. As can be seen from Fig. IA, $D_{p}$ can be changed by changing $h$. The distance $D_{p}$ is called the pivot distance and in this example $D_{p}<D$.

Suppose that for some reason the perceived distance $D_{n}^{\prime}$ of the point of light is less than $D_{p}$. In this case, as shown in Fig. 1A, the point of light will appear to move concomitantly with the head motion through an apparent distance $h^{\prime}$ (between $n_{1}^{\prime}$ and $n_{2}^{\prime}$ ) in the same direction as the motion of the head. On the other hand, if the perceived distance of the point $D^{\prime}{ }_{f}$ is greater than $D_{p}$ the apparent concomitant motion $h^{\prime}$ will be opposite to the head motion (between $f_{1}^{\prime}$ and $f_{2}{ }^{\prime}$ ). It follows from Fig. 1 that:

$$
D^{\prime}=D_{p}\left(K-h^{\prime}\right) / K
$$

In equation 1 it is assumed that the observer correctly senses the distance $K$ through which the head is moved and the change in direction to the point of light $\phi_{T}=\phi_{1}+\phi_{2}$ resulting from the head motion. The value of $h^{\prime}$ in equation $I$ is taken as positive when the apparent concomitant motion is in the direction of the head motion and as negative when it is opposite to that of the head motion.

Figure IB is similar to Fig. 1A except that a physical vertical component of concomitant motion, $V$, is added to the physical horizontal component of concomitant motion, $h$, of the point of light. With the phase of the components of physical motion $h$ and $V$ illustrated in Fig. 1B, the point moves physically at an angle $\alpha$ from the horizontal, between lower left and upper right, as the head moves between Positions 1 and 2. Also as shown, if the perceived distance of the point of light is less than $D_{p}$ (e.g. at $\left.D^{\prime} n\right)$, the point will appear to move at an angle $\alpha^{\prime}$ concomitantly with the head, between lower right and upper left $\left(\alpha^{\prime}>90^{\circ}\right)$. If the perceived distance of the point of light is greater than $D_{p}\left(\right.$ e.g. at $\left.D_{j}^{\prime}\right)$ the point will appear to move concomitantly with the head between lower left and upper right $\left(\alpha^{\prime}<90^{\circ}\right)$. It follows from Fig. 1B that

$$
D^{\prime}=\frac{K D D_{p}}{K D-V D_{p} \cot \alpha^{\prime}}
$$

where $\alpha^{\prime}$ is the slant of the apparent path of motion 


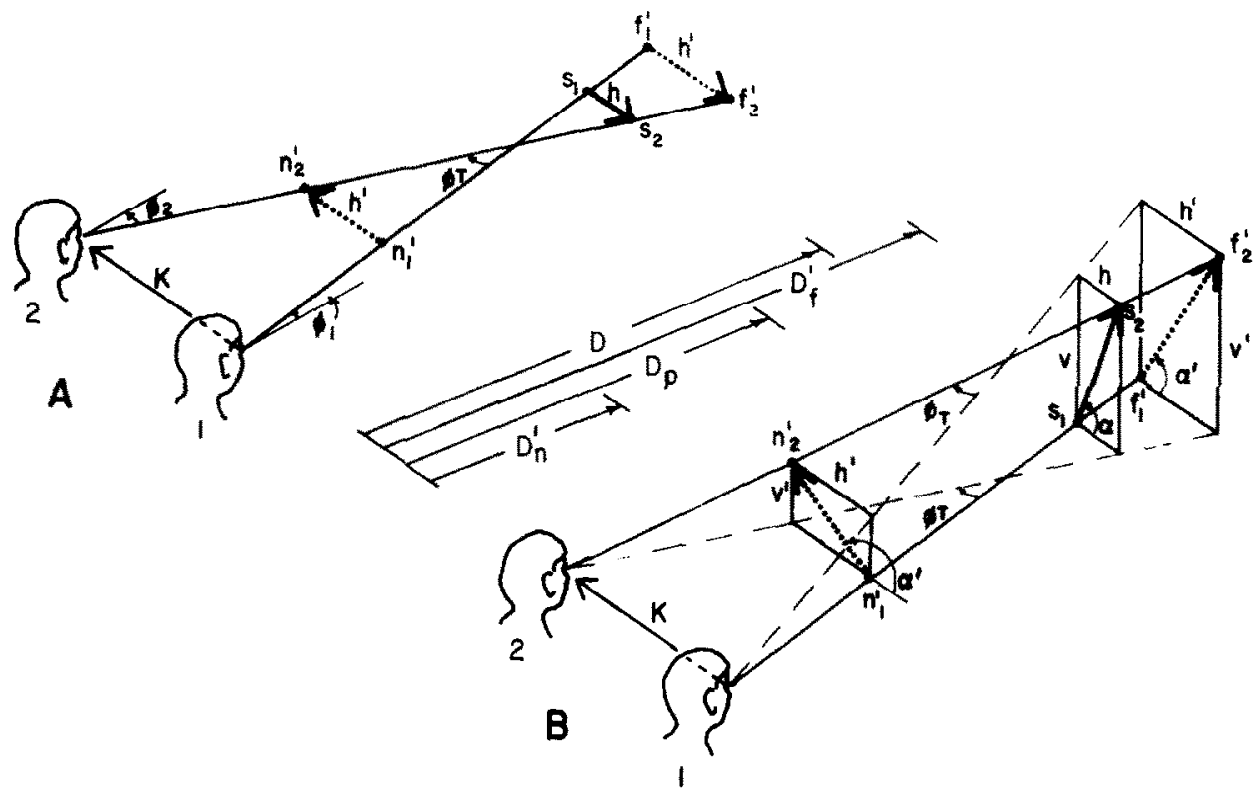

Fig. 1. Perspective drawings illustrating the effect of horizontal head motion upon the apparent horizontal motion $\left(h^{\prime}\right)$ of a point of light as a function of its apparent distance $\left(D^{\prime}\right)$ and pivot distance $(D)$. In $A$, the point physically moves horizontally $(h)$, concumitantly with the motion of the head. In B. a physical vertical motion $(V)$ is added and the concomitant physical motion of the point is at an angle $(\alpha)$.

of the point of light measured counter-clockwise from the horizontal.

Several methods of measuring $D^{\prime}$ follow from equations 1 and 2 . In the situation of Fig. $1 A, D^{\prime}$ can be measured by changing the physical concomitant motion $h$, and thus the magnitude of $D_{p}$, until the point of light no longer appears to move as the head is moved. In this case $h^{\prime}=0$ and according to equation (1), $D_{p}=D^{\prime}$. In the situation of Fig. IB, $D^{\prime}$ can be measured by changing $h$ until the point of light appears to move straight up and down. In this case $x^{\prime}=90^{\circ}$ and, according to equation (2), again $D_{p}=D^{\prime}$. This adjustment of $h$, or equivalently of $D_{p}$, to the criterion of zero apparent horizontal motion (the null criterion) has been the method most frequently used in measuring $D^{\prime}$ in the studies mentioned above. As will be discussed, it is not, however, appropriate for most of the present study. It will be noted that $D_{p}$ specifies the perceived distance expected from the cue of absolute motion parallax, i.e. if absolute motion parallax were totally effective, the point of light regardless of where it was physically, would always appear at $D_{p}$ as the head was moved. The value of $D$ on the other hand specifies the perceived distance expected from oculomotor cues of convergence or accommodation, i.e. if the oculomotor cues were totally effective, the point of light, regardless of the magnitude of $D_{p}$, would always appear at its physical distance $D$ despite the head motion. In order to evaluate the relative effectiveness of these two kinds of cues, the perceived distance of the point must be measured with one cue constant while the other is varied, but this is not possible with the null criterion. Thus, instead of using the null criterion for measuring $D^{\prime}$, in experiments 1 and $2, D^{\prime}$ was obtained by measuring $h^{\prime}$ (Fig. 1A) or $\alpha^{\prime}$ (Fig. 1B) and substituting those into equations (1) and (2), respectively. Experi- ments 1 and 2 of the present study are designed to examine the relative effectiveness of absolute motion parallax and oculomotor cues by determining apparent distance using both $h^{\prime}$ and $\alpha^{\prime}$ measures under conditions in which oculomotor cues and absolute motion parallax are systematically and independently varied. Experiment 3 used the null criterion and examines the major assumption upon which the validity of the head motion procedure is based.

\section{EXPERIMENT 1}

\section{Method}

Obserters. The observers were 4 men and 2 women, 5 of whom were experienced in using the apparatus from their participation in a preliminary experiment. All had a visual acuity of at least $20 / 20$ in both eyes, corrected if necessary, and a stereoscopic acuity of at least $25 \mathrm{sec}$ of arc as measured with the Keystone Orthoscope.

Apparatus. The kind of stimuli presented in experiment 1 is illustrated by Fig. 2. A point of light was presented in an otherwise dark visual field at a near $\left(D_{n}\right)$, middle $\left(D_{m}\right)$, or far $\left(D_{f}\right)$ distance from the observer and was viewed while moving the head repetitively between Positions 1 and 2. At each of these distances $(30.0 \mathrm{~cm}$ in $A, 55.9 \mathrm{~cm}$ in $B$ and $96.4 \mathrm{~cm}$ in $C$ ) the point of light either had zero physical concomitant motion in the horizontal plane or, its horizontal motion, concomitant with the motion of the head, was such as to produce a pivot distance equal to the distance of the point of light when placed at the other two physical distances from the observer. For example. with the point physically at $30 \mathrm{~cm}$ (Fig. 2A) and with the head moving laterally between Positions 1 and 2 , a pivol distance of $30.0 \mathrm{~cm}$ was generated if the horizontal motion of the point was zero $\left(n_{1}\right.$ and $\left.n_{2}\right)$, a pivot distance of $55.9 \mathrm{~cm}$ was generated if the point physically moved between $m_{1}$ and $m_{2}$ and a pivol distance of $96.4 \mathrm{~cm}$ was generated if the point physically moved between $f_{l}$ and $f_{2}$. 
A
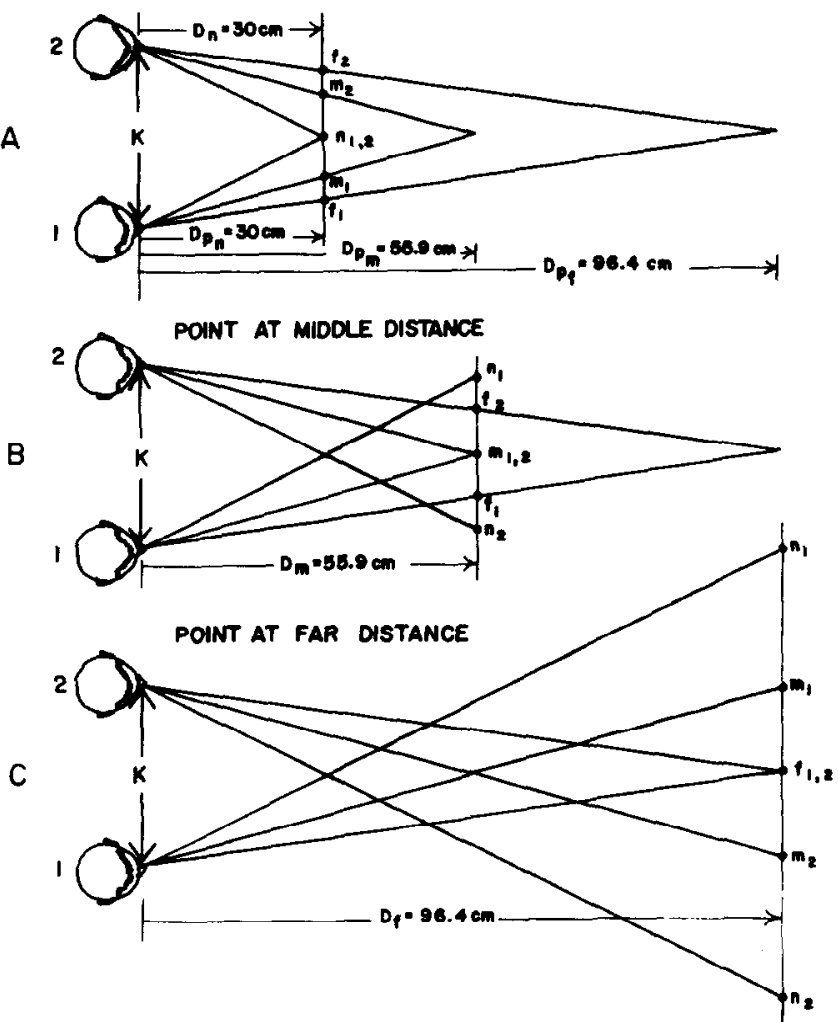

Fig. 2. Top view schematic drawings indicating the physical horizontal motion of a point of light. concomitant with a horizontal motion of the head, required in order to produce three pivot distances $\left(D_{p n}, D_{p m}\right.$ and $\left.D_{p j}\right)$ as a function of the physical distance of the point of light from the observer.

For the conditions in which the observer judged $x$ rather than $h^{\prime}$, a vertical component of physical motion concomitant with the motion of the head was used. This physical motion differed at the near, middle and far distance so as to produce always the same angle of vertical motion on the eyes of the observer. Table 1 shows the magnitude of the component of physical horizontal motion (h) presented with (Fig. 1B) or without (Fig. 1A) the physical vertical component, the magnitude of the physical vertical component $(V)$ when vertical motion was used, and the magnitude of physical slant $(\alpha)$ of the path of motion of the point as measured counter-clockwise from the horizontal, for each combination of physical distance $D$ and pivot distance $D_{p}$ used in the experiment.

The physical concomitant motions of Table 1 were produced by presenting a single point of light on a Conrac
CRT display module (Model CDF). The module was mounted on a track and could be positioned by the experimenter to be at either 30.0 .55 .9 or $96.4 \mathrm{~cm}$ from the observer. The observer sat in a dark observation booth with his head in a head and chin rest and observed the display through an aperature that could be opened or closed by the experimenter. A red acetate filter was placed over the viewing aperture in order to visually eliminate the phosphor trace left by the moving point. The luminous intensity was such that the point was about $1.2 \log$ units above foveal threshold under the adaptation conditions of the experiment. A small shutter attached to the head rest was lowered to occlude the left eye in the monocular conditions and was raised to permit viewing with both eyes in the binocular conditions. Throughout this study nothing was visible during the stimulus presentations except the

Table 1. Physical horizontal motion (h) for the conditions of no vertical motion (Fig. 1A) and components of physical horizontal $(h)^{1}$ and vertical $(V)$ motion for the conditions in which vertical motion was present (Fig. 1B). For the latter conditions, $\alpha$ defines the physical angle of the path along which the point moves; $h$ and $V$ are in centimeters and $\alpha$ is in degrees counter-clockwise from the horizontal. $D$ is the pivot distance and $D$ is the physical distance of the point from the observers

\begin{tabular}{lccccccccc}
\hline & \multicolumn{3}{c}{$D=30.0 \mathrm{~cm}$} & \multicolumn{3}{c}{$D=55.9 \mathrm{~cm}$} & \multicolumn{3}{c}{$D=96.4 \mathrm{~cm}$} \\
\hline$D_{p}$ & $30.0 \mathrm{~cm}$ & $55.9 \mathrm{~cm}$ & $96.4 \mathrm{~cm}$ & $30.0 \mathrm{~cm}$ & $55.9 \mathrm{~cm}$ & $96.4 \mathrm{~cm}$ & $30.0 \mathrm{~cm}$ & $55.9 \mathrm{~cm}$ & $96.4 \mathrm{~cm}$ \\
$h$ & 0.0 & +8.1 & +12.0 & -15.1 & 0.0 & +7.4 & -38.7 & -12.7 & 0.0 \\
$V$ & 6.8 & 6.8 & 6.8 & 12.7 & 12.7 & 12.7 & 21.9 & 21.9 & 21.9 \\
$\alpha$ & 90.0 & 140.0 & 150.6 & 40.0 & 90.0 & 120.1 & 29.5 & 59.7 & 90.0 \\
\hline
\end{tabular}

${ }^{1}$ The plus or minus sign associated with $h$ indicates that the motion of the stimulus point was in the same or opposite direction, respectively, as the motion of the head. The phase of the vertical motion was such that as the head moved to the left the point moved up and as the head moved to the right the point moved down. 
single point of light. The head and chin rest was moveable on ball bearings left and right through a distance of $17.5 \mathrm{~cm}$. To avoid jarring stops, cushioning material was placed at each end of the travel. Depending upon the force exerted by the observer on the head rest, the magnitude of the head motion could differ by $\pm 0.25 \mathrm{~cm}$. The motion of the head between Positions 1 and 2 (see Figs. 1 and 2) was paced by metronome clicks produced over a loudspeaker at the rate of one click every $1.5 \mathrm{sec}$. When the head was at the midpoint of its motion. the stimulus point of light was straight ahead of the observer's right eye and was at eye level. In order to move the point horizontally, vertically or at a slant, concomitant with the head motion, the head rest was geared to a pair of linear potentiometers (one for the horizontal and the other for the vertical component of motion). The potentiometers modulated a voltage as a linear function of the position of the head and chin rest which, upon being applied to the horizontal and vertical plates of the display module, determined the physical motion of the point concomitant with the motion of the head. A control panel, electronically between the display module and the potentiometers, allowed the experimenter to present any of the nine combinations of vertical and horizontal motion components listed in Table 1 .

The observer indicated the perceived slant $\left(x^{\prime}\right)$ or the perceived horizontal motion $\left(h^{t}\right)$ of the point in two ways. These two methods are called the "comparison" and "duplication" method. To indicate the $x^{\prime}$ of the point of light using the comparison method, the observer adjusted the orientation of a rotatable rod $(21 \mathrm{~cm}$ long and $0.4 \mathrm{~cm}$ in diameter) to be the same as the slant of the apparent path of motion of the point. To indicate the $h^{\prime}$ of the point using the comparison method, the observer varied the horizontal separation between two vertical posts $(8 \mathrm{~cm}$ tall and $1.2 \mathrm{~cm}$ in cross section), until their separation was equal to the horizontal motion perceived in the point of light The rotatable rod and adjustable posts were located in the observation booth. The center of the rod was $24 \mathrm{~cm}$ to the right of the viewing aperture and the posts were directly in front of the observer and $44 \mathrm{~cm}$ below eye level. The adjustments of either the rod and posts were read by the experimenter from indicators located at the experimenter's position outside the booth.

For the duplication method of measuring $\alpha^{x}$ or $h^{\prime}$, the head and chin rest was kept stationary by being locked in position at the midpoint of its previous path of motion. Two additions to the apparatus not used with the comparison method were required. One of these consisted of a metal handle $(10 \mathrm{~cm}$ tall and $1.2 \mathrm{~cm}$ in diameter) that the observer, using his left hand, moved left and right in time with the metronome clicks along a track through a horizontal extent of $17.5 \mathrm{~cm}$. The motion of the handle controlled the motion of the point of light in a manner identical to that produced by the previous motion of the head and chin rest. This motion of the handle again varied the voltages from a pair of linear potentiometers as a linear function of the lateral displacement of the handle on the track. These voltages were applied to the horizontal and vertical plates of the display module and caused the point to move. The second addition to the apparatus consisted of a knob located to the right of the observer's position that the observer could turn with his right hand, while moving the handle laterally with his left hand. This knob varied the amount of voltage per unit of lateral motion of the handle which then controlled the horizontal motion of the point on the display module. The vertical motion of the point when present was unaffected by the knob adjustment. Thus by simultaneously moving the handle and adjusting the knob, the observer could vary the physical motion of the point to perceptually duplicate an apparent slant or apparent horizontal motion presented a moment before. The experimenter recorded the observer's knob adjustment from the reading of a digital voltmeter which, when calibrated. indicated the horizontal motion of the point on the display.

Procedure. The observer was given practice moving his head smoothly in the head rest in time with the metronome clicks. The duplication and comparison methods were explained and the observer was cautioned to respond on the basis of the apparent motion of the point of light regardless of whether this was thought to be the same or different from its physical motion. The instructions were to directly fixate the point of light at all times. A single trial proceeded as follows: the booth lights were extinguished, the metronome was turned on and, after the observer began moving his head, the stimulus was presented. The observer watched the point moving until the amount of apparent horizontal motion (horizontal condition) or the apparent slant (slant condition) of the point was firmly in mind. The shutter was then closed and the observer indicated the apparent slant or horizontal extent using either the comparison or duplication method. For the duplication method the head and chin rest was moved to the central position where the experimenter locked it in place. The observer then moved the handle left and right in time with the metronome, the shutter was opened. and the observer rotated the knob until the point appeared to be moving exactly as it had appeared to move in the immediately preceding trial with the head motion. The shutter was then closed and the booth lights were turned on between trials. For the comparison method, following the presentation of the point stimulus, the shutter was closed and the booth lights turned on. The observer removed his head from the head and chin rest and adjusted either the rotatable rod or separable posts to indicate the perceived slant or perceived horizontal extent of motion of the point, respectively. With both the duplication and comparison methods the observer was instructed to use a bracketing technique whereby the final adjustment was reached following adjustments to either side of the desired setting using successively smaller deviations.

Following the presentation of a trial in which $D$ and $D_{p}$ were the same, the observer was asked to report verbally the perceived distance of the point in feet or inches or some combination of both. Between all trials, with the booth lights on, the observer was light adapted for $15 \mathrm{sec}$. by looking at the center of a large white sheet of cardboard with a luminance of $11.0 \mathrm{~cd} / \mathrm{m}^{2}$

All observers received all nine conditions specified in Table I (three physical distances of the display with three values of $D_{p}$ at each physical distance). All three values of $D_{p}$ were presented at one distance before presenting the next distance, with the same order of $D_{p}$ values maintained at each display distance. The order of presenting the three values on $D_{p}$ and $D$ were determined by a balanced Latin square. The order of presenting the slant $(\alpha)$ or horizontal (h) conditions, the order of monocular or binocular viewing, and the order in which the duplication and comparison methods were used was systematically varied between observers.

\section{Results}

The results from experiment 1 are summarized in Fig. 3. The $D^{\prime}$ data of Fig. 3 were obtained by applying equations (1) and (2) to the $h^{\prime}$ and $\alpha^{\prime}$ results averaged over the six observers for each of the particular combinations of conditions indicated in the figure. The average verbal reports of distance of the point of light (converted to centimeters) when $D$ and $D_{p}$ were both at the near, middle, and far distances were 49,75 , and $118 \mathrm{~cm}$, respectively.

Oculomotor cues. If oculomotor cues were effective in modifying perceived distance, $D^{\prime}$ would increase with $D$, i.e. the curves of Fig. 3 would slope upward. 


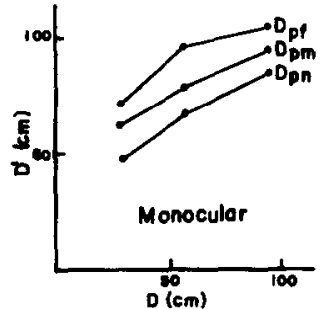

HORIZONTAL COMPARISON

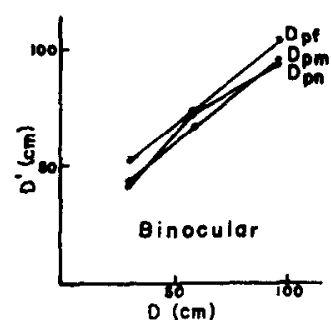

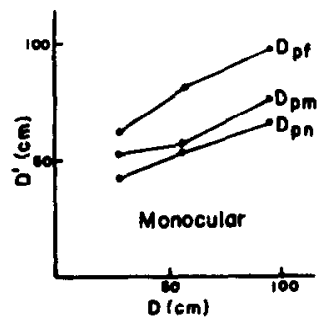

SLANT COMPARISON

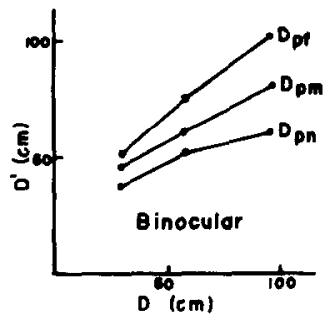

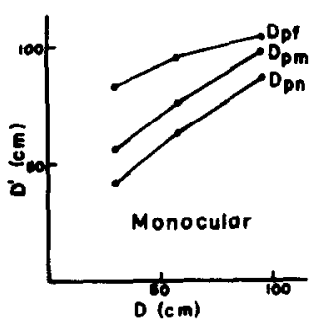

HORIZONTAL DUPLICATION

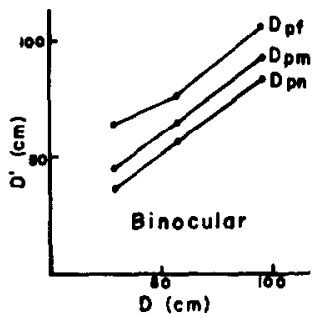

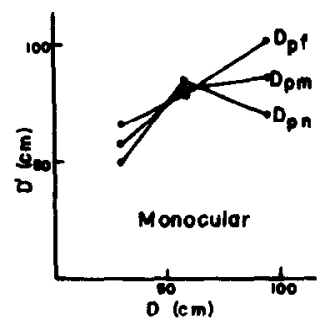

SLANT DUPLICATION

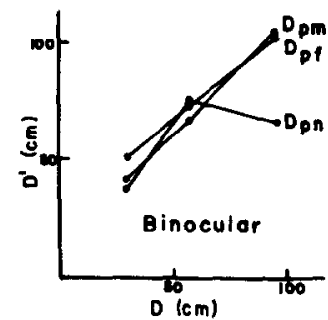

Fig. 3. The relation between perceived distance $\left(D^{\prime}\right)$ and physical distance $(D)$ of a point of light in Experiment 1 for three pivot distances $\left(D_{p}\right)$, using the comparison or duplication methods with either horizontal motion $(h)$ only or a slanted motion $(\alpha)$ of the point, with two modes of observation (monocular or binocular).

As can be seen this is generally the case for both monocular and binocular observation using the data from either the horizontal $(h)$ or slant $(\alpha)$ conditions with both the comparison and duplication method. According to the analysis of variance, $D$ was a significant determiner of $D^{\prime}, F(2,4)=382.0, P<0.01$. Also, the average $D^{\prime}$ from binocular observation $(68.9 \mathrm{~cm})$ and monocular observation $(74.3 \mathrm{~cm})$ differed significantly, $F(1,4)=23.9, P<0.01$.
A summary of the $D^{\prime}$ data of Fig. 3 as a function of $D$ averaged over the three values of $D_{p}$ and over the comparison and duplication methods is shown in the two drawings on the left half of Fig. 4. If oculomotor cues had completely dominated the perception despite the constant average value of $D_{p}$, the curves would slope at $45^{\circ}$ (the dashed lines) and if totally ineffective the curves would be horizontal. Monocular and binocular observation provided somewhat differ-

\section{Slant Condition}
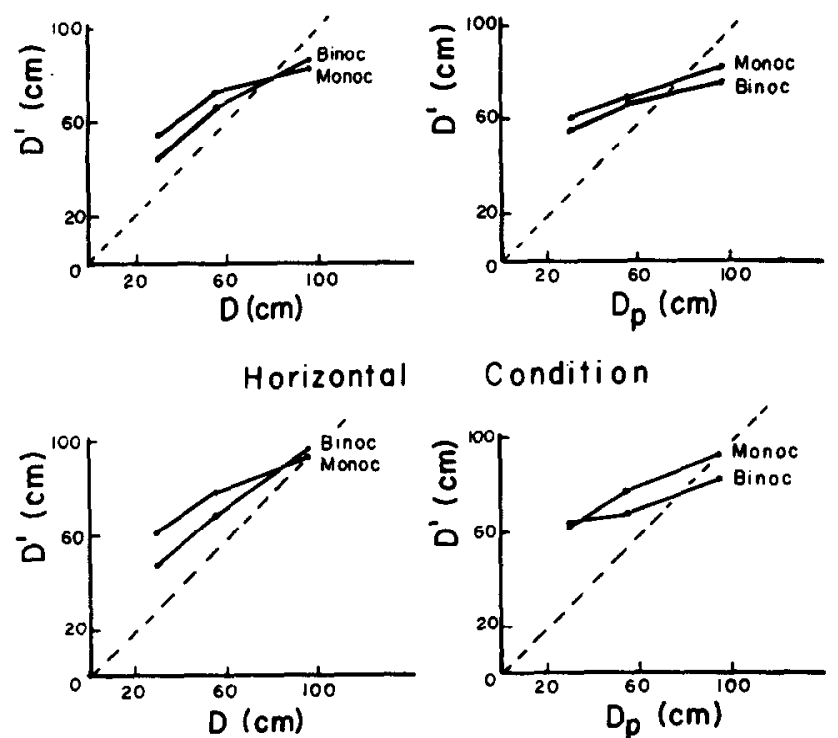

\section{Condition}

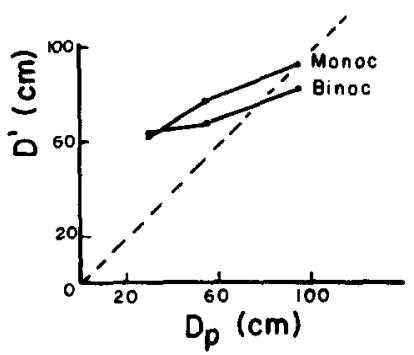

Fig. 4. Summary of results from Experiment 1. In all four graphs the data points are averages of results from the comparison and duplication methods. The graphs on the left show the change in $D^{\prime}$ as a function of $D$ (oculomotor cues) averaged over the three values of $D_{p}$ (absolute motion parallax). The graphs on the right show the change in $D^{\prime}$ as a function as $D_{p}$ averaged over the three values of $D$. 
ent oculomotor cues. With monocular observation the oculomotor cues consisted of accommodation and the convergence associated with accommodation (accommodative convergence). With binocular observation accommodation and fusional convergence were available. It was expected that binocular observation would be more effective than monocular observation in providing cues of distance (Gogel and Sturm, 1972). Consistent with this expectation, the slope of the curves relating $D$ and $D^{\prime}$ in Fig. 4 is greater with binocular as compared with monocular observation. This is in agreement with the analysis of variance in which the interaction of physical distance and mode of observation was statistically significant $F(2,4)=16.3, P<0.05$. Also, Fig. 4 indicates that the measurement of $D^{\prime}$ by $h^{\prime}$ and by $x^{\prime}$ gives very similar results.

Absolute motion parallax. With either monocular or binocular observation the distance cue of absolute motion parallax was determined by $D_{p}$. If absolute motion parallax had been totally effective, the point of light would have always appeared at $D_{p}$. That absolute motion parallax was somewhat effective is indicated in Fig. 3 by the tendency for the $D_{p n}$ curve to be lowest and the $D_{p f}$ curve highest on the graphs. According to the analysis of variance, absolute motion parallax was a significant cue to distance, $F(2,4)=123.5, P<0.01$. The drawings on the right half of Fig. 4 graph the perceived distance data of Fig. 3 as a function of $D_{p}$ averaged over the three values of $D$ and over the comparison and duplication methods. If monocular parallax had completely dominated the perception despite the constant average value of $D$, the curves would slope at $45^{\circ}$ (the dashed lines) and if totally ineffective the curves would be horizontal. Consistent with the greater effectiveness of oculomotor cues from binocular than monocular observation, the slope of these curves should be less using binocular observation.

Several other factors also were statistically significant in the $D^{\prime}$ data shown in Fig. 3. The interaction of type of motion and method of measurement was significant, $F(1,4)=17.7, P<0.05$, with the average $D^{\prime}$ less from using $\alpha$ with the comparison method $(64.5 \mathrm{~cm})$ than from using $x$ with the duplication method (73.9). The average $D^{\prime}$ obtained using the comparison method $(69.7 \mathrm{~cm})$ differed significantly from that obtained using the duplication method $(73.4 \mathrm{~cm}), F(1,4)=9.9, P<0.05$. Also, the mean $D^{\prime}$ obtained from $h^{r}(74.4 \mathrm{~cm})$ was significantly different from that obtained from $\alpha^{\prime}(68.6 \mathrm{~cm}), F(1.4)-27.1$ $P<0.01$. As will be seen, however, this latter difference is not supported by the results of experiment 2. Finally, the interaction of $D_{p}$, node of observation, and type of stimulus motion ( $\alpha$ or $h$ ) also was signifcant $F(2,4)=10.4, P<0.05$. Although the obtained value of $D^{\prime}$ can differ for the two methods of measurement and perhaps for the two types of stimulus motion, the effect of these factors on the computed value of $D^{\prime}$ is relatively minor. The apparent concomitant motion of the point of light that occurs with the head motion is, of course, independent of the method by which this apparent motion is measured. Methods in addition to the comparison or duplication method could have been used. It was thought prior to the experiment that the duplication method would be particularly appropriate since this method reproduced exactly the motion perceived during the experimental presentations. This expectation, however, received no encouragement from Fig. 3 since the least consistent combination of method of measurement and type of motion seems to have been the duplication method with $x$.

Comparison of oculomotor and absolute motion parallax cues. The data directly relevant to comparing the effectiveness of oculomotor cues and cues of absolute motion parallax are shown in Fig. 5. Depending on the combination of values of $D$ and $D_{p}$, oculomotor cues and absolute motion parallax were in agreement or were opposed in determining $D^{\prime}$. Cue agreement occurred when $D$ and $D_{p}$ were the same distance (i.e. $D_{n} D_{p n}, D_{m} D_{p m}$, or $D_{f} D_{p f}$ ). Opposition of cues occurred when $D$ and $D_{p}$ were different distances (i.e. $D_{n} D_{p f}$ or $D_{f} D_{p n}$ ). The results from these different conditions averaged over all observers and type of motion $(h$ or $\alpha$ ) is shown in the two graphs of Fig. 5 . with the condition $D_{m} D_{p m}$ appearing in both graphs. Of particular interest are the results from the opposition of cues. If the oculomotor cues had been completely effective despite the opposed absolute motion parallax, the curves would be parallel to the dashed line. If the cue of absolute motion parallax had been completely effective despite the opposed oculomotor cues, the curves would be orthogonal to the dashed line. If the oculomotor cues and absolute motion parallax were equally effective the data curves would be horizontal. Since this latter case occurred with monocular observation, it follows that oculomotor cues from monocular observation and the cue of absolute motion parallax were about equally effective. The

CUES AGREE

CUES OPPOSED
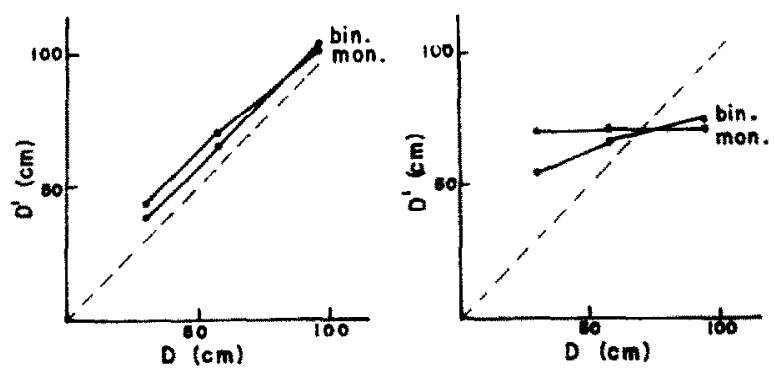

Fig. 5. The relation between perceived distance $\left(D^{\prime}\right)$ and physical distance $(D)$ of a point of light in Experiment 1 as a function of whether oculomotor and absolute motion parallax cues of distance are in agreement or disagreement, using two modes of observation (monocular or binocular). 

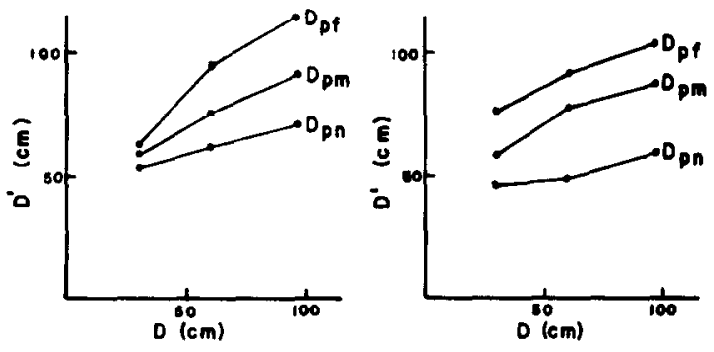

HORIZONTAL CONDITION

\section{SLANT} CONDITION
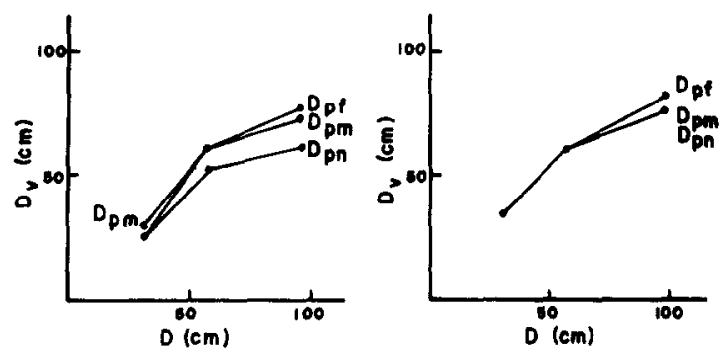

Fig. 6. The relation between physical distance $(D)$ and perceived distance measured by the head motion procedure $\left(D^{\prime}\right)$ or by verbal reports $\left(D_{v}\right)$ in Experiment 2 , using the comparison method and monocular observation of the point of light.

upward slope of the "binocular" curve on the other hand indicates that oculomotor cues from binocular observation were more effective than the cue of absolute motion parallax.

\section{Discussion}

From Figs 3 and 4 it is clear that both oculomotor cues and absolute motion parallax were effective in modifying perceived distance. According to Fig. 5, absolute motion parallax was about equal in effectiveness to the oculomotor cues from monocular observation, but both the absolute motion parallax cue and oculomotor cues from monocular observation were less effective than cues from binocular observation. It should be noted also in the left drawing of Fig. 5 that even with oculomotor cues and absolute motion parallax in agreement, the perception of distance was not veridical, i.e. $D^{\prime}$ was greater than $D$ (or $D_{p}$ ). This is consistent with the modification of $D^{\prime}$ from oculomotor cues and absolute motion parallax by the specific distance tendency (Gogel, 1977; Gogel and Tietz, 1973). The specific distance tendency is the tendency in the absence of effective cues to distance to perceive objects at a distance of several meters. With distance cues somewhat reduced, as in the present study, the perceived distance is a compromise between these cues and the perceived distance indicated by the specific distance tendency.

\section{EXPERIMENT 2}

Experiment 2 was similar to experiment 1 except that only monocular observation and the comparison method were used. The purpose of experiment 2 was to determine whether naive observers would show results similar to those obtained in experiment 1 , and whether the effect of oculomotor and absolute motion parallax cues on $D^{\prime}$ would be reflected in verbal reports of distance as well as in the measures obtained with the head motion procedure.

\section{Method}

Observers. The observers were 36 undergraduate psychology students, 19 men and 17 women, with an average age of 18 years who partially satisfied a course requirement by participating in the experiment. All were unaware of the purpose of the experiment and all had at least $20 / 20$ uncorrected vision both near and far in the right eye.

Apparatus. The apparatus was identical to that of experiment 1 except that the portion of the apparatus required for the duplication method was not used.

Procedure. The procedure was identical to that used with the monocular conditions of experiment 1 . Only the comparison method of measuring $h^{\prime}$ and $x^{\prime}$ was used with the addition that at the beginning of every trial, after at least $S$ left-right movements of the head, the observer reported the apparent distance to the point of light, in feet or inches or some combination of both, while continuing to move his head. As in experiment 1 both the horizontal $(h)$ and slant motions of the point of light $(\alpha)$ were included.

\section{Results}

The head motion procedure. The average values of $h^{\prime}$ and $\alpha^{\prime}$ converted to $D^{\prime}$ by equations (1) and (2) for the measures obtained from the head motion procedure are shown in the upper drawings of Fig. 6. From the analysis of variance, the only statistically significant factors were $D$ and $D$, with $F(2,4)=62.6$, $P<0.01$ and $F(2,4)=84.2, P<0.01$, respectively. The effect of $D$ (accommodation and accommodative convergence) on $D^{\prime}$ is similar to that obtained from the same condition in experiment 1 , with the only clear difference being that the overall average $D^{\prime}$ from $\alpha^{\prime}(76.5 \mathrm{~cm})$ was slightly greater (not less) than that from $h^{\prime}(71.9 \mathrm{~cm})$. As in experiment 1 , both the oculo- 
motor cues from monocular observation and absolute motion parallax were effective in modifying $D$. When these cues were in agreement, the change in $D^{*}$ was from $52.6 \mathrm{~cm}\left(D_{n} D_{p n}\right)$ to $111.2 \mathrm{~cm}\left(D_{f} D_{p f}\right)$ whereas, when opposed, the change was from $73.6 \mathrm{~cm}\left(D_{n} D_{p f}\right)$ to $85.0 \mathrm{~cm}\left(D_{f} D_{p n}\right)$. Again, this indicates that the effectiveness of absolute motion parallax as a distance cue is similar to although possibly slightly less than that of accommodation.

Verbal reports. The median values of reported distance $\left(D_{v}\right)$ as a function of $D$ are shown in the lower portion of Fig. 6. Medians rather than means are used because the distributions of verbal reports tend to be skewed. It is evident from the upward slope of these curves that the verbal reports of distance increased systematically with increasing physical distance. Thus, $D^{\prime}$ as measured by either the head motion procedure or by verbal reports of distance in experiment 2 clearly changed as a function of the accommodative cue of distance. The results from the verbal reports do not as clearly support the conclusion that absolute motion parallax is an effective cue to distance. The curves in the lower graphs of Fig. 6 are not clearly separated as a function of $D_{p}$, particularly for the slant $(\alpha)$ condition. A Friedman rank order analysis was applied to the $D_{v}$ data. The increase in reported distance with increasing $D$ was significant for each of the six curves of the lower portion of Fig. $6 ; \chi^{2}=31.2,42.8$ and $42.7, \mathrm{df}=2, P<0.01$ for the horizontal condition and $\chi^{2}=44.0,39.2$ and $39.2, \mathrm{df}=2, p<0.01$ for the slant condition. However, only in the case of the horizontal condition with $D=96.4 \mathrm{~cm}$ was the increase in the verbal report of distance with increasing $D_{p}$ significant; $\chi^{2}=7.1$, $\mathrm{d} f=2, P<0.05$. Unlike the measures of $D^{\prime}$ obtained from the head motion procedure, there is only weak evidence that absolute motion parallax modified the verbal reports of distance. Possibly this can be understood as a consequence of the high variability of verbal reports of distance. It should be noted, however, that the oculomotor cues of distance from the monocular obscrvation, which, according to the results from the head motion procedure, are not much more effective than absolute motion parallax, were clearly able to modify the verbal reports of distance.

\section{Discussion}

The difference in the effectiveness of absolute motion parallax as a cue to perceived distance measured by the head motion procedure and by verbal reports of distance can be explained in two ways. One explanation is that, although motion parallax modifies perceived distance, this modification is not reflected in the verbal report of distance. A second possibility is that motion parallax is not an effective cue but instead the angle $\phi_{T}$ (see Fig. 1) at the pivot distance is not correctly sensed by the observer. Suppose, for example, that the error in estimating $\phi_{T}$ varies with the magnitude of $\phi_{T}$ so that small values of $\phi_{T}$ are overestimated relative to larger values. This misperception of $\phi_{T}$ would result in a change in $h^{\prime}$ in the direction such that $D^{\prime}$, as calculated from $h^{\prime}$ using equation 1 , would be an overestimation of the $D^{\prime}$ actually perceived. Thus, an increase in $D_{p}$ would increase the value of $D^{\prime}$ calculated from equation (1) or equation (2), even though $D^{\prime}$ actually remained constant. This second possible explanation was tested in experiment 3 in which the extent of horizontal head motion $(K)$ and thus, the magnitude of $\phi_{T}$ was varied systematically.

\section{EXPERIMENT 3}

The null criterion discussed above was used in experiment 3 to measure the perceived distance of the point of light at a constant physical distance by adjusting the path of apparent motion of the point $\left(\alpha^{\prime}\right)$ until the point appeared to move straight up and down $\left(\alpha^{\prime}=90^{\circ}\right)$. Under these conditions $\cot \alpha^{\prime}=0$ and from equation $2, D^{\prime}=D_{p}$. The null criterion was used with different amounts of head motion $(K)$ so as to produce different values of $\phi_{T}$. If the $D_{p}$ (or $D^{\prime}$ ) obtained in this manner is the same for all values of $K$, it follows that the effect of $D_{p}$ on $D^{\prime}$ in experiments 1 and 2 using the head motion procedure cannot be attributed to misperceptions of $\phi_{T}$ as a function of $D_{p}$ and, therefore, must be attributed to absolute motion parallax as a cue to distance.

\section{Method}

Observers. The same observers were used in experiment 3 as had been used in experiment 1 .

Apparatus. The head and chin rest assembly for the lateral head motion used in the previous experiments was modified in construction and use for experiment 3 as follows. Instead of observing the point of light while moving the head both right and left, the point was seen only when the head was moved from right to left. This procedure avoided the need to stop the motion of the head abruptly at either end of its travel. In a preliminary study, such abrupt stops although of little concern with large head motions were found to produce a disturbing bounce in the perceived motion of the point for small values of $K$. The starting position for the head rest was the stop at the right which, together with the position of a microswitch as explained below, provided three extents of head motion $(5.4 \mathrm{~cm}, 9.4 \mathrm{~cm}$, or $17.5 \mathrm{~cm})$. symmetrical about the center of the viewing aperture. As the head rest moved toward the left a microswitch which turned off the point of light was positioned so that it was activated by the head rest when the head had moved through one of the three distances specified above. The point of light remained extinguished until the head rest was returned to the right position where a second microswitch again turned on the point. Thus, the observer saw the point of light only when his head was moving left and only during the motion of his head between the right stop and the microswitch on the left.

In order to indicate the desired speed of head motion to the observer, a Hunter timer was triggered when the head rest moved from the right stop and after a preset time interval, a buzzer sounded. The observer was in structed to move the head in the left direction at a velocity which would cause the head rest to actuate the left microswitch (extinguish the point of light) simultaneously with the sounding of the buzzer. Three different time intervals were used corresponding to the three extents of head motion so as to produce a constant head velocity of $11.6 \mathrm{~cm} / \mathrm{sec}$ in all conditions. An elapsed time clock was started when the head rest began its left to right motion and was stopped when the left microswitch was activated thereby allowing the experimenter to calculate the average head velocity of the observer.

The stimulus was the single point of light at $30.0 \mathrm{~cm}$ used previously. The vertical component of motion always was $0.39 \mathrm{~cm}$ per $\mathrm{cm}$ of head motion. The horizontal component was varied by the knob on the observer's right 
so that the apparent slant of the path of motion of the point could be adjusted to the apparent vertical. The physical slant adjusted on the display module by the observer in order to achieve apparently vertical motion in the point was determined from the reading on the digital voltmeter located at the position of the experimenter.

Procedure. The observer was given practice with this new head motion procedure before beginning the experiment. The instructions were to he id the head stationary at the right stop until the point of light was clearly fixated and then to move the head from right to left at the constant velocity sufficient to cause the point to disappear simultaneously with the sound of the buzzer. The observer's task was to use the knob near the observation position to adjust the direction of motion of the point of light until the point appeared to move straight up as the head moved left. If during a head movement the point appeared to move at a slant the observer made an adjustment of the knob followed by another head movement. with the knob always adjusted between head movements. The bracketing technique again was used but with the added procedure that the final adjustment was reached from opposite directions (clockwise or counter-clockwise) on successive trials. When the observer was satisfied that the point appeared to move vertically, the experimenter recorded the knob adjustment and the total time for the head movements during that trial. The average velocity of head motion was calculated by dividing the product of $K$ and the number of head movements by the summed time during which the observer moved his head to the left between the microswitches. Observers typically required about eight head movements to complete a single adjustment of the point of light to the apparent vertical.

Each observer completed 12 adjustments (trials) to the apparent vertical for each of the three extents $(K)$ of head motion. The 12 trials at a given value of $K$ were completed before changing to a different value. The order of the three values of $K\left(K_{s}=5.4, K_{m}=9.4\right.$, and $\left.K_{L}=17.5 \mathrm{~cm}\right)$ was counterbalanced between observers. After every six trials the observer rested and was light adapted by looking at the white surface for $1 \mathrm{~min}$ with the booth light on. The observation was always monocular using the right eye only.

\section{Results}

The relation between $K$ and $D^{\prime}$ (or $D_{p}$ ) is given in Table 2 for the six observers. The effect of size of $K$ on $D^{\prime}$ was not found to be statistically significant using the analysis of variance, $F(2,10)=1.26$, $P>0.05$. The average velocity of motion of the head was $10.0,10.9$ and $11.0 \mathrm{~cm} / \mathrm{sec}$ for $K_{s}, K_{m}$ and $K_{L}$, respectively, with these values not greatly different from the intended value of $11.6 \mathrm{~cm} / \mathrm{sec}$. It seems from these results that the effect of absolute motion parallax on $D^{\prime}$ in experiment 1 cannot be attributed to a nonlinear relation between the physical and perceived size of $\phi_{T}$.

Discussion. The values of $K$ used in experiment 3 would have produced values of $\phi_{T}$ identical to those of experiments 1 and 2 if $D^{\prime}$, and thus $D_{p}$ with the null criterion, had been equal to $D$. Because of the specific distance tendency, however, $D^{\prime}$ and $D_{p}$ were greater than $D$. The values of $\phi_{T}$, computed from the means of Table 2 , were $2.45^{\circ}, 4.35^{\circ}$ and $8.83^{\circ}$ for $K_{s}$ $K_{m}$, and $K_{L}$, respectively. The values of $\phi_{T}$ used in experiments 1 and 2 were $5.18^{\circ}, 8.90^{\circ}$, and $16.27^{\circ}$ for $D_{p L}, D_{p m}$ and $D_{p s}$ respectively. Although the changes in $\phi_{T}$ used in experiment 3 were less extensive than those in experiments 1 and 2, it is clear from experiment 3 that despite substantial changes in $\phi_{T}$ pro-
Table 2. Perceived distance in centimeters of the point of light as measured by the head motion procedure, using three values of extent of head motion $(K)$ and the criterion of adjusting the motion of the point until it appeared to move vertically

\begin{tabular}{cccc}
\hline Observer & $K_{s}$ & $K_{\mathrm{m}}$ & $K_{L}$ \\
\hline 1 & 38.0 & 38.4 & 42.2 \\
2 & 39.4 & 41.0 & 45.8 \\
3 & 78.9 & 74.8 & 67.1 \\
4 & 64.4 & 70.2 & 62.2 \\
5 & 63.3 & 59.3 & 59.7 \\
6 & 95.8 & 87.4 & 60.6 \\
Mean & 63.3 & 61.8 & 56.3 \\
Median & 63.9 & 64.8 & 60.2 \\
SD & 22.4 & 19.4 & 9.9 \\
\hline
\end{tabular}

duced by changes in $K, D^{\prime}$ as measured by the null criterion did not substantially change. Experiment 3 could have been performed with equal and constant values of $D$ and $D_{p}$, with either the comparison or duplication method to measure $\alpha^{\prime}$ for the computation of $D^{\prime}$, using the three values of $K$. Such a test would be appropriate for experiment 3 only if it could be assumed that the effectiveness of absolute motion parallax was the same for all three head motions. Evidence regarding this is not available. As can be inferred from the discussion to follow, the null criterion does not involve this assumption.

Experiment 3 provides evidence that systematic errors in the sensing of $\phi_{r}$ do not occur as a function of the size of $\phi_{T}$. It follows that the effect of absolute motion parallax on $D^{\prime}$ in experiments 1 and 2 cannot be explained by errors in the perception of direction as a function of the magnitude of $D_{p}$. It also follows from the present experiment that verbal reports of distance do not readily reflect the perception of distance from absolute motion parallax even though they clearly reflect the perception of distance from oculomotor cues. Perhaps some cognitive effects, presently unidentified, intrude on the verbal reports to limit their usefulness here as in the case with familiar objects (Gogel, 1976).

\section{CONCLUDING REMARKS}

From the results of this study, both oculomotor cues and absolute motion parallax contribute to perceived distance. Of the oculomotor cues, those from monocular observation are less effective than those from binocular observation, and absolute motion parallax is only slightly less effective than the former, but considerably less effective than the latter. The contribution of absolute motion parallax to perceived distance, although clearly significant as measured by the head motion procedure, is only very marginally significant as measured by verbal reports of distance.

It was expected that oculomotor cues from binocular observation would modify perceived distance (Foley \& Held, 1972; Gogel, 1977). The change in perceived distance as a function of the physical distance of the point using monocular observation is surprising, however, since according to Owens and Leibowitz (1975), a dim point of light is not an adequate stimulus for accommodation (or accommodative con- 
vergence). Perhaps retinal blur rather than a response of the ciliary muscle provided the crucial distance information. Except for studies by Ferris (1972) and by Johansson (1973) there has been little evidence that motion parallax is an effective cue to egocentric distance. Perhaps one reason for the difficulty in detecting its effect on apparent distance is that this effect is not readily evident in verbal reports of distance. It should be noted, however, that absolute motion parallax must be regarded as a rather weak cue to distance since its contribution in the present study was at least cancelled by equal but opposite changes in accommodation.

The data of the present study suggest that the head motion procedure can provide a sensitive and valid measure of perceived distance. Throughout this study. the observers were instructed to fixate carefully the point of light. The reason for this, of course, was to insure that the occulomotor cues would be appropriate to the physical distance of the point. From their own observations, the experimenters have found that relaxing fixation slightly will be reflected immediately in a change in the apparent path of motion of the point of light viewed with the moving head. If supported by more formal observations, such a result would reflect very favorably upon the sensitivity of the head motion procedure of measuring apparent distance.

Basically, there are two tasks that have been used for measuring perceived distance with the head motion procedure. One, the null criterion, measures $D^{\prime}$ by having the observer adjust $D_{p}$ to remove all apparent horizontal motion concomitant with the horizontal motion of the head. If no vertical motion is present in the stimulus the adjustments to this criterion will result in the stimulus appearing stationary $\left(h^{\prime}=0\right)$. If vertical concomitant motion is present, the null criterion consists of adjusting the path of motion of the stimulus until it appears to be moving vertically $\left(\alpha^{\prime}=90^{\circ}\right)$. The adjustment of $D_{p}$ until $h^{\prime}=0$ or $\alpha^{\prime}=$ $90^{\circ}$ removes the possibility that absolute motion parallax will influence the measurement of $D^{\prime}$. The reason for this is that, with the null criterion, $D_{p}$ (the absolute motion parallax cue) is adjusted until it is in agreement with the perceived distance of the object as determined by other distance cues. Suppose, for example, that the null criterion is used to measure $D^{\prime}$ from convergence by varying $D_{p}$ until $\alpha^{\prime}=90^{\circ}$. In this case, whenever $D_{p}$ is different from $D$, the value of $D^{\prime}$ will be modified to some extent by the absolute motion parallax defined by $D_{p}$. However, $\alpha^{\prime}$ will not equal $90^{\circ}$ until the absolute motion cue from $D_{p}$ is adjusted to be in agreement with $D^{\prime}$ from the cue of convergence. If the bracketing technique as applied in experiment 3 or some similar method is used to counterbalance the direction in which $D_{p}$ is adjusted to the null criterion, the cue of absolute motion parallax associated with $D_{p}$ will not introduce a mean error in the measurement of the perceived distance of the stimulus object.

The second task that can be used to measure perceived distance with the head motion procedure is that of indicating $h^{\prime}$ or $\alpha^{\prime}$ as in experiments 1 and
2 of the present study. This task although useful in these experments, is not generally preferred, since, unlike the null criterion, the measured value of $D^{\prime}$ of the stimulus using this task can be modified (as shown in the present study) by the distance cue of absolute motion parallax. As in the case of the null criterion. the measurement of $D^{\prime}$ with $h^{\prime} \neq 0$ or $x^{\prime} \neq 90^{\circ}$ provides a valid, unbiased measure of the perceived distance of the stimulus. It is a perceived distance, however, that is likely to have been at least partly determined (and in experiments 1 and 2 the effect is appreciable) by the distance cue of absolute motion parallax.

In the beginning of this article, it was noted that previous studies clearly have shown that $D^{\prime}$ as measured using the null criterion is at least a monotonic increasing function of perceived distance. From the results of experiment 3 , it seems, with this method. that the measured value of $D^{\prime}$ indeed equals the perceived distance of the stimulus. The use of the head motion procedure particularly with the null criterion seems to provide a measure of the perceived distance of a stimulus that is valid, sensitive, and free from the intrusion of cognitive factors.

Acknowledgements-We thank Bernard W. Griffin for his assistance in collecting the data of Experiment 2. This work was supported by the National Science Foundation under Grant BNS 77-16620.

\section{REFERENCES}

Ferris S. H. (1972) Motion parallax and absolute distance. J. exp. Psychol. 95, 258-263.

Foley J. M. and Held R. (1972) Visually directed pointing as a function of target distance, direction and available cues. Percept. Psychophys. 12, 263-268.

Gogel W. C. (1974) Cognitive factors in spatial responses. Psychologia 17, 213-225.

Gogel W. C. (1976) An indirect method of measuring perceived distance from familiar size. Percept. Psychophys. 20, 419-429.

Gogel W. C. (1977) An indirect measure of perceived distance from oculomotor cues. Percept. Psychophys. 21, 3-11.

Gogel W. C. and Newton R. E. (1976) An apparatus for indirect measurement of perceived distance. Percept. Mot. Skills 43, 295-302.

Gogel W. C. and Sturm R. D. (1976) A comparison of accommodative and fusional convergence as cues to distance. Percept. Psychophys. 11, 166-168.

Gogel W. C. and Tietz J. D. (1973) Absolute motion parallax and the specific distance tendency. Percept. Psychophys. 13, 284-292.

Gogel W. C. and Tietz J. D. (1974) The effect of perceived distance on perceived movement. Percept. Psychophys. 16, 70.78 .

Gogel W. C. and Tietz J. D. (1977) Eye fixation and attention as modifiers of perceived distance. Percept. Mot. Skills 45, 343-362.

Johansson G. (1973) Monocular movement parallax and near space perception. Perception 2, 135-146.

Owens D. A. and Leibowitz H. W. (1975) The fixation point as a stimulus for accommodation. Vision Res. 15. 1161-1163. 